\title{
The c-Jun signaling pathway has a protective effect on nucleus pulposus cells in patients with intervertebral disc degeneration
}

\author{
MING LEI, KUN WANG, SHUAI LI, KANGCHENG ZHAO, WENBIN HUA, XINGHUO WU and CAO YANG \\ Department of Orthopaedics, Union Hospital, Tongji Medical College, \\ Huazhong University of Science and Technology, Wuhan, Hubei 430022, P.R. China
}

Received March 7, 2020; Accepted July 1, 2020

DOI: $10.3892 /$ etm.2020.9251

\begin{abstract}
Among a range of diverse clinical symptoms, intervertebral disc degeneration (IDD) contributes mostly to the onset of lower back pain. The present study aimed to investigate the effects of c-Jun on nucleus pulposus (NP) cells of IDD and its regulation on molecular mechanisms. Intervertebral disc (IVD) tissues were collected from patients suffering from IDD disease, and NP cells were subsequently isolated and cultured. By overexpressing c-Jun in NP cells, expression levels of $\mathrm{mRNAs}$ and proteins of IDD-related genes and inflammatory cytokines were subjected to reverse transcription-quantitative PCR, western blot and ELISA assays. Additional transforming growth factor- $\beta$ (TGF- $\beta$ ) antibodies were administrated to suppress the function of TGF- $\beta$. Cell proliferation and apoptosis were determined via Cell Counting Kit-8 and TUNEL assays, respectively. The results demonstrated that the overexpression of c-Jun robustly upregulated both mRNA and protein expression of TGF- $\beta$, TIMP metallopeptidase inhibitor 3, aggrecan and collagen type II alpha 1 chain and simultaneously downregulated the expression of the inflammatory cytokines TNF- $\alpha$, interleukin (IL)-1 $\beta$, IL-6 and IL-17. Furthermore, following c-Jun overexpression, survival rates of NP cells were increased while apoptosis rates were decreased. However, the addition of a TGF- $\beta$ antibody significantly promoted apoptosis and restricted cell survival, which differed from the results of the c-Jun overexpression group. The present study hypothesized therefore that c-Jun may positively regulate TGF- $\beta$ expression within NP cells of IDD, which could promote the proliferation of IDD-NP cells and accelerate cell viability via reducing apoptosis and the inflammatory response.
\end{abstract}

Correspondence to: Professor Cao Yang, Department of Orthopaedics, Union Hospital, Tongji Medical College, Huazhong University of Science and Technology, 1277 Jiefang Avenue, Wuhan, Hubei 430022, P.R. China

E-mail: caoyang0278@foxmail.com

Key words: c-Jun, intervertebral disc degeneration, nucleus pulposus, transforming growth factor- $\beta$, inflammatory cytokines

\section{Introduction}

The intervertebral disc (IVD) is composed of nucleus pulposus (NP), anulus fibrosus and cartilage endplate (1). Back pain is mostly attributable to the degeneration of IVD, which may cause nerve damage, poor labor capacity and low quality of life in some severe cases (1). Despite intervertebral disc degeneration (IDD) development being a natural process that comes with aging, some evidence suggests that the development of IDD stems from both genetic and environmental factors (2). For example, certain factors, including the decrease of NP cells, imbalance between the synthesis and degradation of the extracellular matrix (ECM) and overexpression of inflammatory cytokines, are known to be responsible for accelerating the development of IDD (3).

NP cell-produced collagen type II alpha 1 chain (COL2A1), aggrecan (ACAN) and some ECM components are believed to be crucial to maintain the integrity of IVD $(4,5)$. It has been reported that some molecules, such as insulin-like growth factor-1, epidermal growth factor and transforming growth factor- $\beta$ (TGF- $\beta$ ), can promote anabolic metabolism of ECM (6,7). Tumor necrosis factor (TNF)- $\alpha$, interleukins (IL), matrix metalloproteinases (MMPs) and a disintegrin and metalloproteinase with thrombospondin type I motifs (ADAMTS) work synergistically and facilitate the catabolism of the ECM (8-10). Catabolic activities of MMPs and ADAMTSs were leveraged by inhibitory actions and through tissue inhibitors of metalloproteinases (TIMPs) (10). In NP cells, TGF- $\beta$ can delay the process of IDD via promoting the synthesis of ACAN and COL2A1, which enhances cell proliferation and inhibits MMPs expression $(6,11)$. IDD NP cells also produced diverse inflammatory mediators which caused the aggravation of IDD, such as TNF- $\alpha$, IL-1 $\alpha / \beta$, IL-6 and IL-17 (12).

As a major component of activator protein-1 (AP-1), c-Jun expression is increased when cells enter the logarithmic phase of growth in the presence of growth-factor-induced stimulation (13). Wisdom et al (14) demonstrated that c-Jun stimulates fibroblast growth and inhibits its apoptosis. Furthermore, c-Jun was found to protect cells from TNF- $\alpha$-induced apoptosis, which is required for cell proliferation (14). Conversely, the presence and phosphorylation-based activation of c-Jun are necessary for the execution of apoptosis in both neuronal cells and thymocytes (15). In the intestinal ischemia-reperfusion injured autograft model, activation of both c-Fos and c-Jun genes can trigger cell proliferation and apoptosis (16). 
Behrens et al (17) reported that a lack of c-Jun in mice can lead to the impairment of hepatocyte proliferation and liver regeneration. Notochordal cells in the absence of c-Jun have also been demonstrated to experience an increase in apoptosis, leading to impairment of IVD formation (18). Subsequently, it was speculated that c-Jun expression may have an essential role in IDD process. However, the function and underlying mechanisms of c-Jun in IDD remain unknown. Because the present study aimed to explore the role of c-Jun in NP cells, NP cells were transduced with a c-Jun-overexpressing lentivirus, and changes in IVD-related genes on a molecular level were detected. This study attempted to further elucidate the pathogenesis of IDD and provide a novel addition to valuable clinical information for the treatment of IDD.

\section{Materials and methods}

IVD tissue collection. IVD tissues were collected as surgical waste from 10 patients with IDD (age, 35-58 years). In addition, IVD tissues from 10 patients with lumbar fractures (age, 26-52 years), excluding those with spinal tumors, infections and rheumatic immune diseases, were collected as controls. This study was approved by the Institutional Review Board of Tongji Medical College and followed the Declaration of Helsinki. Written informed consent was obtained from each patient. According to the MRI scanning techniques reported by Pfirrmann et al (19), the obtained IVD tissues were graded by $\mathrm{T} 2$-weighted images to determine degrees of degeneration. Relative normal nondegenerated discs from patients with lumbar fractures were graded I-II (Control), whereas degenerative discs from patients with IDD were graded III-V. Subsequently, NP cells were isolated from IVD tissues of patients with IDD and control subjects.

Isolation and culture NP cells. Primary NP cells were isolated and cultured as previously reported (5). The IDD and control NP tissue samples were washed three times with D-Hanks solution under aseptic conditions. These specimens were cut into $1-\mathrm{mm}^{3}$ pieces, and digested with $0.25 \%$ trypsin (Beyotime Institute of Biotechnology) and $0.2 \%$ collagenase II (Beyotime Institute of Biotechnology) for $3 \mathrm{~h}$ at $37^{\circ} \mathrm{C}$. NP cells were filtered through a 200-mesh sieve, washed three times with PBS and the supernatant was discarded following centrifugation at $2,000 \mathrm{x}$ g for $5 \mathrm{~min}\left(37^{\circ} \mathrm{C}\right)$. Cells were washed with DMEM-F12 (Gibco; Thermo Fisher Scientific, Inc.) medium containing $10 \%$ FBS to terminate digestion. After centrifugation at $2,000 \mathrm{x} \mathrm{g}$ for $5 \mathrm{~min}\left(37^{\circ} \mathrm{C}\right)$, NP cells were counted and seeded into $25 \mathrm{~cm}^{2}$ culture dishes. DMEM-F12 medium was supplemented with 15\% FBS (Gibco; Thermo Fisher Scientific, Inc.), $10 \mu \mathrm{g} / \mathrm{ml}$ insulin and $1 \%$ penicillin/streptomycin (Invitrogen; Thermo Fisher Scientific, Inc.) was used to culture NP cells under conventional incubation conditions $\left(37^{\circ} \mathrm{C}, 5 \% \mathrm{CO}_{2}\right.$ and $95 \%$ humidity), and the medium was refreshed twice a week. When NP confluency reached $80 \%$, cells were passaged at a ratio of 1:2. Cells in passage P2 were used for subsequent experiments.

Lentiviral vector construction and lentivirus infection of $N P$ cells. The c-Jun gene was inserted into green fluorescence protein (GFP)-labeled LV5 plasmids obtained from
Boaimaidisen Biotechnology Co., Ltd. and the lentivirus was packaged by four plasmid systems namely LV5-c-Jun, PG-p1-VSVG, PG-P2-REV and PG-P3-RRE (Boaimaidisen Biotechnology Co., Ltd.). Transfections were performed into 293 T packaging cell lines (American Type Culture Collection). Lentiviral packaging enrichment was completed by Chongqing Biomedicine Biotechnology Co., Ltd. The GFP-labeled blank LV5-empty vector was used as the negative control. The viral titer of LV5-c-Jun and LV5-empty vector lentivirus was $1 \times 10^{8} \mathrm{TU} / \mathrm{ml}$.

Before lentiviral transfection, a preliminary test was carried out to estimate the efficiency of lentiviral infection in target cells. The multiplicity of infection (MOI) refers to the proportion of infectious viruses per cells and is calculated as follows: $\mathrm{MOI}=($ virus titer $\mathrm{x}$ virus volume)/number of cells. NP cells were seeded into 24-well culture plate at the density of $3 \times 10^{4} /$ well. $293 \mathrm{~T}$ cells served as the control cells in a parallel experiment to determine the affinity of lentivirus to target cells. Transfection was conducted when the confluency reached $70 \%$. The infectious lentiviruses were incubated with the NP cells and control cells at a final MOI of 0,10 , 20, 40 and 100. The mixed lentivirus and cells were incubated in a incubator $\left(37^{\circ} \mathrm{C}, 5 \% \mathrm{CO}_{2}\right)$ overnight. After $24 \mathrm{~h}$, culture medium containing lentivirus was replaced with normal culture medium. At 4 days following infection, the transduction efficiency was tested via flow cytometry according to GFP-positive cells (BD Diagnostics). GFP expression was observed in cellSens 1.12 software (Olympus Corporation) at a magnification of x100 using an inverted fluorescence microscope (CKX53, Olympus Corporation).

Western blotting. NP cells were lysed in RIPA reagent (Beyotime Institute of Biotechnology), and the concentration of extracted proteins was measured by BCA standard method (Takara Biotechnology Co., Ltd.). Proteins $(30 \mu \mathrm{g})$ were separated by $10 \%$ SDS-PAGE and transferred onto PVDF membranes (EMD Millipore). PVDF membranes were washed with TBST $(0.05 \%$ Tween-20) three times before being blocked with $5 \%$ skimmed milk at room temperature for $2 \mathrm{~h}$. Primary antibodies (1:500) were incubated overnight at $4^{\circ} \mathrm{C}$. Subsequently, the HRP-conjugated goat anti-rabbit antibody was incubated for $1.5 \mathrm{~h}$ at room temperature $(1: 1,500$, ab205718; Abcam). The proteins were visualized by chemiluminescence (Thermo Fisher Scientific, Inc.,). Densitometry was semi-quantified with ImageJ 1.8.0 software (National Institutes of Health). Results were normalized by GAPDH. The following primary antibodies were used: Anti-c-Jun (cat. no. ab40766), anti-TGF- $\beta$ (cat. no. ab31013), anti-TIMP-1 (cat. no. ab211926), anti-TIMP-3 (cat. no. ab39184), anti-ADAMTS-4 (cat. no. ab185722), anti-ADAMTS-5 (cat. no. ab41037), anti-ACAN (cat. no. ab3778), anti-COL1A1 (cat. no. ab34710), anti-COL2A1 (cat. no. ab34712), anti-IL-1 $\beta$ (cat. no. ab2105), anti-IL-6 (cat. no. ab6672), anti-IL-17 (cat. no. ab79056), anti-TNF- $\alpha$ (cat. no. ab6671), anti-GAPDH (cat. no. ab181602) (all from Abcam).

Reverse transcription-quantitative PCR (RT-qPCR) assay. Total RNA was isolated from NP cells using TRIzol ${ }^{\circledR}$ reagent (Beyotime Institute of Biotechnology) according to the manufacturer's instructions. Reverse transcription was carried out 
using the PrimeScript ${ }^{\mathrm{TM}}$ RT reagent kit with gDNA Eraser (Takara Bio, Inc.), according to the manufacturer's instructions. The reverse transcription reactions occurred as follows: $37^{\circ} \mathrm{C}$ for $15 \mathrm{~min}, 85^{\circ} \mathrm{C}$ for $5 \mathrm{sec}, 4^{\circ} \mathrm{C}$ for $5 \mathrm{~min}$. RT-qPCR analysis was performed on an ABI 7500 instrument (Thermo Fisher Scientific, Inc.). All reaction systems and procedures of RT-qPCR were conducted following the manufacturer's protocol of TB Green Premix Ex Taq II (Takara Bio, Inc.). Reaction protocol was set with three-step cycling conditions: $95^{\circ} \mathrm{C}$ for $30 \mathrm{sec}$, followed by 40 cycles of $95^{\circ} \mathrm{C}$ for $5 \mathrm{sec}, 60^{\circ} \mathrm{C}$ for $30 \mathrm{sec}$ and $72^{\circ} \mathrm{C}$ for $15 \mathrm{sec}$. The melting curve stage was included, ramping from 65 to $95^{\circ} \mathrm{C}$ (increment $0.5^{\circ} \mathrm{C} / 5 \mathrm{sec}$ ) to verify the specificity of the primer amplification based on the presence of a single and sharp peak. All primer sequences are listed in Table SI. The $2^{-\Delta \Delta \mathrm{Cq}}$ method was used to calculate the relative expression levels (20).

ELISA assay. After the c-Jun- and empty vector-transfected NP cells were cultured, the supernatants were collected into sterile centrifuge tube. The expression levels of inflammatory factors, including TNF- $\alpha$ (cat. no. P01P0087; Shanghai BlueGene Biotech Co.,Ltd.), IL-1 $\beta$ (cat.no.E01I0010; Shanghai BlueGene Biotech Co., Ltd.), IL-6 (cat. no. E01I0006; Shanghai BlueGene Biotech Co., Ltd.) and IL-17 (cat. no. E01I0362; Shanghai BlueGene Biotech Co., Ltd.) were measured by ELISA according to the manufacturer's instructions.

Cell Counting Kit-8 (CCK8) and TUNEL assay. According to the CCK-8 (Sigma-Aldrich; Merck KGaA) manufacturer's instructions, $100 \mu \mathrm{l} /$ well cell suspension was seeded into a 96 -well plate $\left(1 \times 10^{4}\right.$ cells/well). After being cultured for $24 \mathrm{~h}$ at $37^{\circ} \mathrm{C}$, c-Jun-mediated cells were treated with TGF- $\beta$ neutralizing antibody $(2 \mu \mathrm{g} / \mathrm{ml}$; cat. no. AB-100-NA; $\mathrm{R} \& \mathrm{D}$ Systems, Inc.) and isotype-matched control IgG (2 $\mu \mathrm{g} / \mathrm{ml}$; cat. no. AB-108-C; R\&D Systems, Inc.). Upon $12 \mathrm{~h}$ of incubation, $10 \mu \mathrm{l}$ CCK8 solution was added to each well and for $2 \mathrm{~h}$. The absorbance was measured at $450 \mathrm{~nm}$ on a microplate reader.

For the TUNEL assay, cells were fixed with $4 \%$ paraformaldehyde for $20 \mathrm{~min}$ at room temperature. Cells were washed three times with PBS and cells were stained according to the manufacturer's instructions of the TUNEL kit [cat. no. 11684817910; Roche Diagnostics (Shanghai) Co., Ltd.]. Apoptotic cell was observed at a magnification of x100 using an inverted fluorescence microscope (cat. no. CKX53; Olympus Corporation), each sample was randomly counted in four fields, with red representing apoptotic cells and blue representing nucleus pulposus. The apoptosis rate was the percentage of red fluorescent cells to blue fluorescent cells, and the average value was taken as the final apoptosis rate of nucleus pulposus cells.

Statistical analysis. Data were presented as the means \pm standard deviation. Each experiment was repeated three times independently. Statistical analyses were performed using SPSS 23.0 (IBM Corp.). Comparisons among multiple groups were made with one-way analysis of variance (ANOVA) followed by a Tukey's post hoc test and differences between two groups were identified with a Student's t-test. $\mathrm{P}<0.05$ was considered to indicate a statistically significant difference.

\section{Results}

Cell isolation and lentivirus transfection. The present study demonstrated that NP cells were successfully isolated from both normal and IDD tissues. Briefly, following c-Jun overexpression, GFP expression in normal NP cells displayed 25, 63, 78 and $93 \%$ efficiencies at MOI values of 10, 20, 40 and 100, respectively. When MOI values were set at 10, 20, 40 and 100, the GFP expression efficiency in the IDD NP cells exhibited $26,47,74$ and $88 \%$, respectively. These results indicated that cell expressed the transfected GFP most efficiently when the MOI value was set at 100 (Fig. 1A and B).

Detection of c-Jun expression by western blotting and $R T-q P C R$. The results from western blotting indicated that c-Jun expression was the highest when the MOI was set at 100 for both normal and IDD NP cells (Fig. 1C and D). A similar increase in c-Jun expression was observed by RT-qPCR between the normal and IDD groups (Fig. 1E). These results demonstrated a successful overexpression of c-Jun in NP cells. Notably, the data demonstrated that c-Jun expression was lower in IDD NP cells compared with normal NP cells (Fig. 1C-E).

c-Jun overexpression upregulates the genes associated with ameliorating IDD. The expression levels of IDD-related factors were detected by western blotting and RT-qPCR following transduction (MOI $100 \mathrm{PFU} /$ cell) and subsequent cell culture for 3 and 7 days in the normal and IDD NP cells. On day 3, a comparison was made between the c-Jun-overexpressed IDD NP cells and the empty vector-transfected cells. The results demonstrated that c-Jun-overexpression groups exhibited significant increases in the mRNA expression of TGF- $\beta$, TIMP-3, ADAMTS-4, ACAN and COL2A1, and a significant decrease in COL1A1 expression, while no significant changes were detected in TIMP-1 and ADAMTS-5 expression (Fig. 2A). On day 7, expression levels of TGF- $\beta$, ACAN and COL2A1 were significantly upregulated after c-Jun overexpression when compared with the control. The effect of c-Jun on NP cells was most evident on the third day; consequently, the cells transfected for 3 days were selected for subsequent protein detection (Fig. $2 \mathrm{~B}$ and $\mathrm{C}$ ). The results suggested that expression levels of TGF- $\beta$, TIMP-3, ACAN and COL2A1 were significantly increased after c-Jun overexpression in the degenerative NP cells. Taken together, these data demonstrated that c-Jun overexpression may promote the expression of proteins associated with IDD amelioration.

c-Jun overexpression downregulates the expression of inflammatory cytokines. The expression levels of TNF- $\alpha$, IL-1 $\beta$, IL- 6 and IL-17 were detected by RT-qPCR, western blotting and ELISA. The results indicated a significant decrease in mRNA and protein expression of TNF- $\alpha$, IL-1 $\beta$, IL- 6 and IL-17 following c-Jun overexpression in normal and IDD NP cells (Figs. 2B, 3A-E). In addition, the expression levels of inflammatory cytokines in IDD NP cells were higher than those in normal healthy NP cells. Similar concentration-dependent trends were observed in the ELISA assays. Furthermore, both in normal and IDD NP cells, the concentration of inflammatory factors was significantly lower than those in the empty-vector transfected cells following c-Jun overexpression (Fig. 3F-I). 

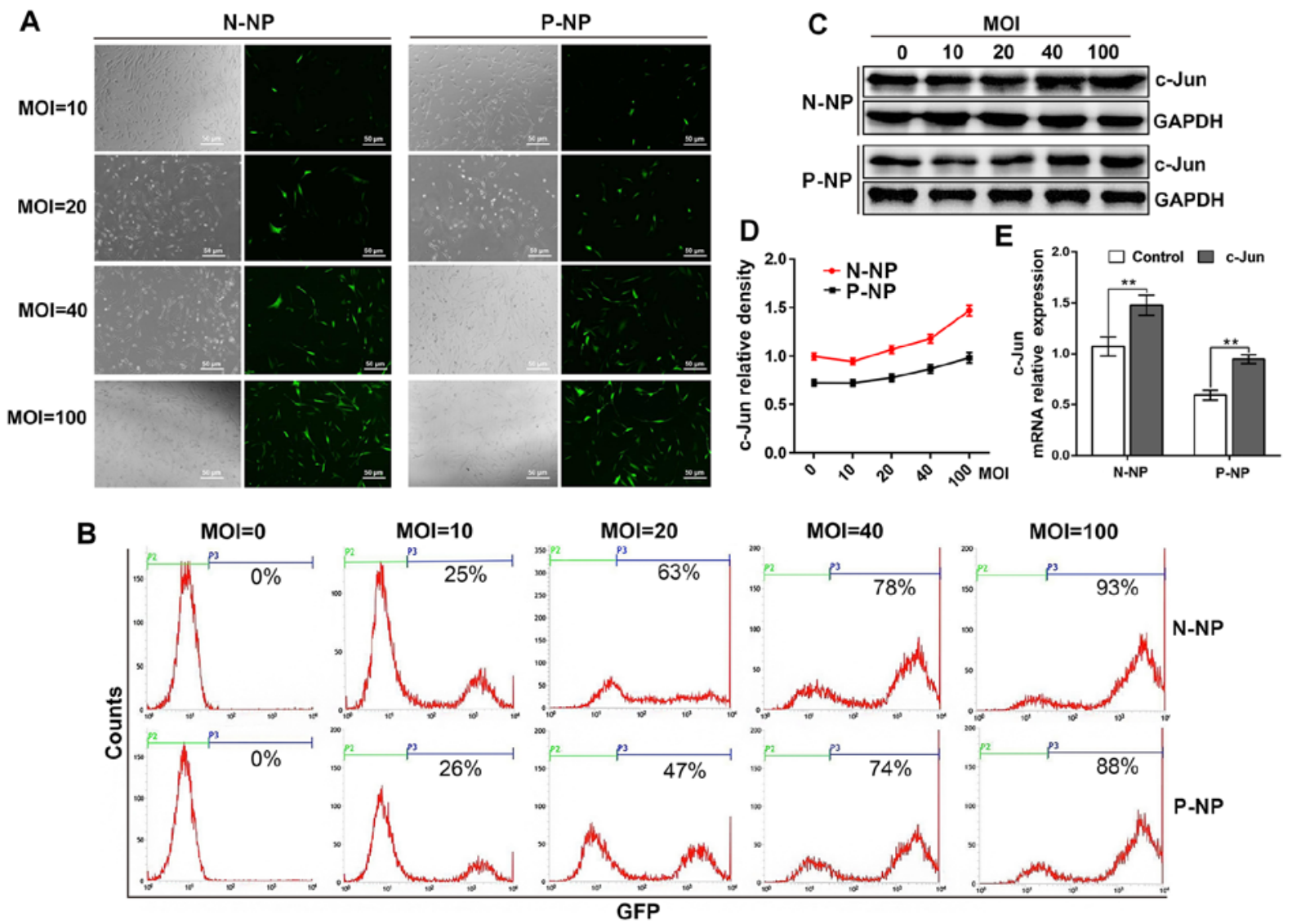

Figure 1.c-Jun was successfully overexpressed in normal and degenerative NP cells. (A) Efficiency of lentiviral infection at four different MOI. (B) Efficiency of lentivirus infection was detected by flow cytometry. (C) Protein expression of c-Jun was detected by western blotting. (D) c-Jun relative density in (B) was analyzed by ImageJ. (E) The mRNA expression of c-Jun at MOI=100 was detected by reverse transcription-quantitative PCR. ${ }^{* *} \mathrm{P}<0.01$. Scale bar, $50 \mu \mathrm{m}$. Control, empty vector-transfected group; c-Jun, overexpressing c-Jun group; MOI, multiplicity of infection; N-NP, normal nucleus pulposus cells; $\mathrm{P}-\mathrm{NP}$, degenerative nucleus pulposus cells.

These findings suggested that the overexpression of c-Jun inhibited the expressions of inflammatory cytokines.

Effects of TGF- $\beta$ antibody on c-Jun-regulated cell. A previous study reported that c-Jun can activate TGF- $\beta$-induced transcription (21). To clarify the roles of TGF- $\beta$ in c-Jun-regulated cell proliferation and apoptosis, cells were treated with TGF- $\beta$ antibody to inhibit TGF- $\beta$ activity, and the the survival and apoptosis rates of degenerated cells were analyzed by CCK8 and TUNEL assays, respectively. Survival rates in the empty vector-transfected and the c-Jun transfected cells were 100 and $103.15 \%$, respectively. The survival rates manifested differently following the addition of TGF- $\beta$ antibody, which significantly decreased cell survival to $86.42 \%$, compared with $103.28 \%$ in the c-Jun + IgG1 group (Fig. 4A). These results indicated that TGF- $\beta$ antibody was capable of decreasing cell proliferation and inducing cell death in c-Jun-overexpressed NP cells. Consequently, the apoptosis rates of empty vector-transfected group, c-Jun transfected group, c-Jun + TGF- $\beta$ antibody group and c-Jun + IgG1 control group were evaluated, and the results demonstrated that the apoptosis rates were 8.15, 7.15, 21.25 and $5.48 \%$, respectively (Fig. 4B and C). The apoptosis rates increased significantly following TGF- $\beta$ antibody addition, indicating that TGF- $\beta$ could inhibit the apoptosis following c-Jun overexpression within IDD NP cells.

\section{Discussion}

As a member of the AP-1 transcription factor family, c-Jun has a functional role in various cellular responses to extracellular stimuli $(13,22)$. By functioning as a key transcription factor, AP-1 was reported to regulate cell survival and cell death pathways, initiate gene transcription as a molecular switch, and was demonstrated to be involved in relevant cellular processes including proliferation, apoptosis and inflammation (22). In c-Jun-deficient mice, the IVD formation was impaired (18). In addition, IVD cell clusters produce certain ECM components, allowing the repair of damaged tissues with c-Jun as a major protein expressed (23). It was therefore hypothesized that c-Jun could have crucial effects on IVD tissues. However, the role of c-Jun in the progression of IDD remains unknown, in particular the its effects on NP cell proliferation, apoptosis and target gene regulation in vitro. The results from the present study demonstrated that the mRNA and protein expression of c-Jun in normal NP cells was higher compared with that in IDD NP cells. Furthermore, c-Jun had a positive effect on NP cells in promoting cell proliferation, reducing cell apoptosis, increasing ECM synthesis and downregulating inflammatory factors.

A previous study demonstrated that inflammatory mediators serve crucial roles in IVD and could contribute to the IDD process (24). The expression levels of inflammatory factors IL-1 $\beta$, IL-6, IL-17 and TNF- $\alpha$ in the degenerated 
A

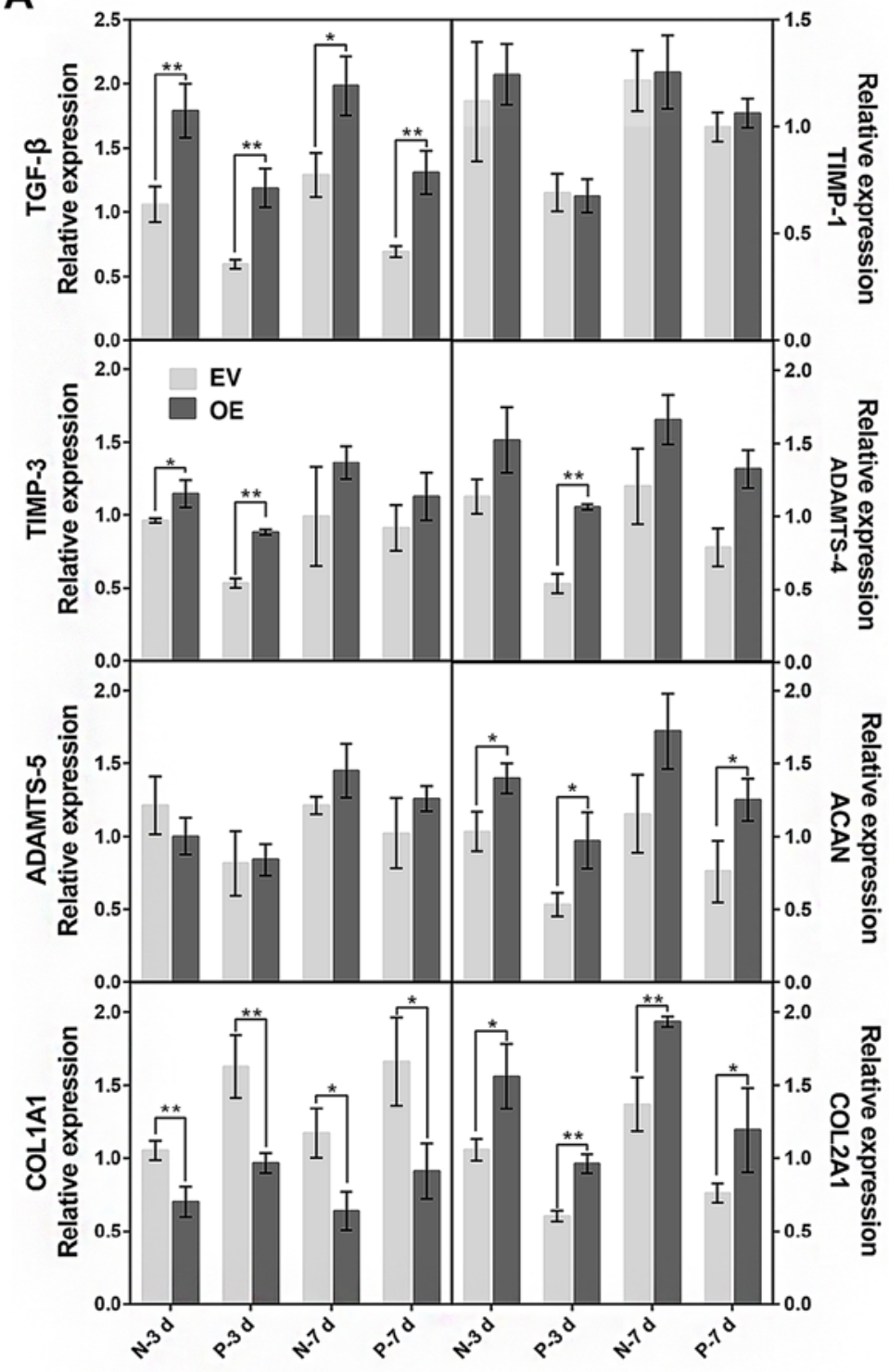

B

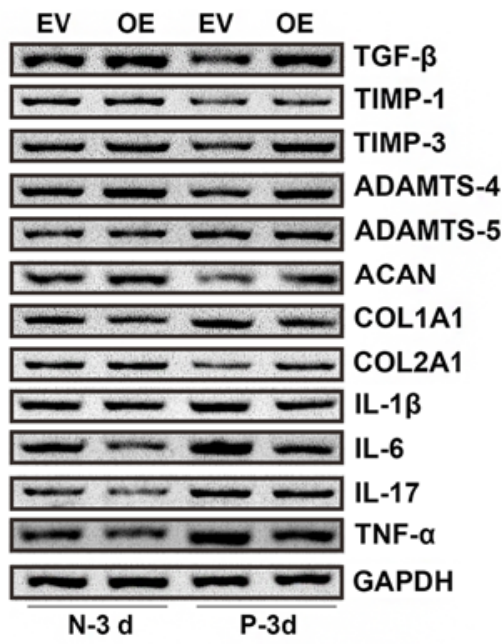

C

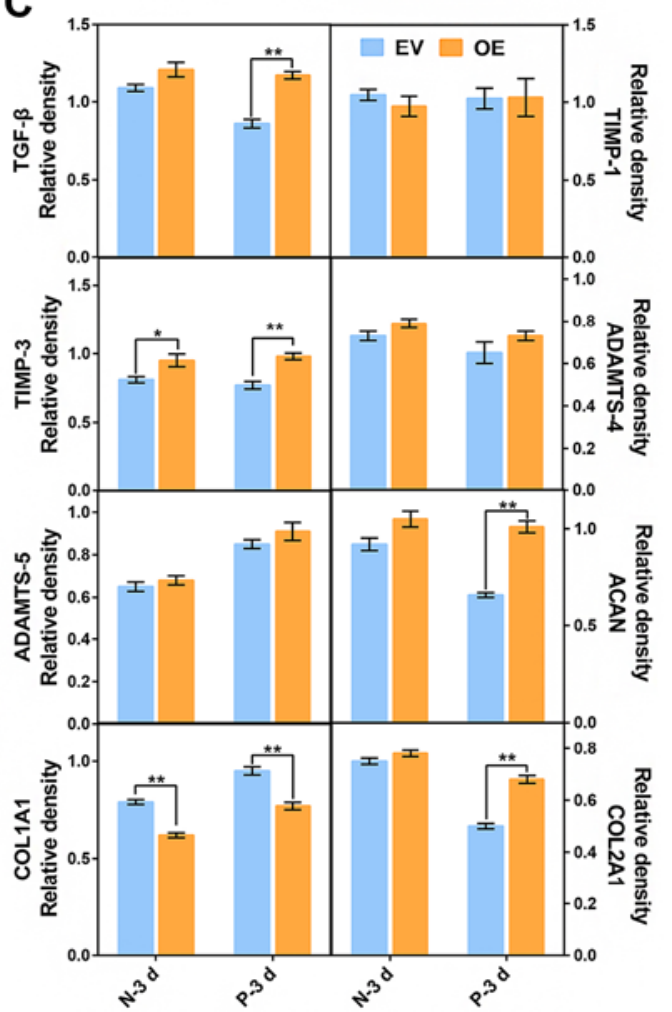

Figure 2. Expression of genes associated with synthesis and catabolism in NP cells following c-Jun overexpression. (A) mRNA expression of genes associated with anabolism in NP cells was detected by reverse transcription-quantitative PCR after cells being cultured for 3 and 7 days. (B) Protein expression of these genes was detected by western blotting after cells being cultured for 3 days. (C) Relative density in (B) was analyzed by ImageJ. ${ }^{*} \mathrm{P}<0.05$ and ${ }^{* *} \mathrm{P}<0.01$. EV, empty vector-transfected cells; OE, c-Jun-overexpressed cells; N-3 d, -7 d, normal cells were cultured for 3 days and 7 days; D-3 d, $-7 \mathrm{~d}$, degenerative cells were cultured for 3 days and 7 days.

disc tissue were distinctly increased compared with normal disc tissue, and their expressions was positively correlated with the degree of disc degeneration, including IL-1 $\beta$, IL-6, IL-17 and TNF- $\alpha$ (25). In addition, AP-1 is considered as a pro-inflammatory factor that can directly regulate the expression of ILs and MMPs (26). The results from the present study suggested that NP cells overexpressin c-Jun exhibited a dramatic decrease in the expression of TNF- $\alpha$, IL- $1 \beta$, IL- 6 and IL-17, suggesting that c-Jun may exert an anti-inflammatory function in NP cells. However, AP-1 dimers composed of different protein subunits processed different biological functions, and Jun family members exhibit different functional properties as transcription factors (27). Li et al (28) reported that IL-17a can enhance the expression of cyclooxygenase 2 and prostaglandin E2 in NP cells, leading to the regulation of inflammatory responses through p38/c-Fos and c-Jun n-terminal kinase (JNK)/c-Jun pathways. Duval et al (29) reported that the JNK pathway could downregulate the production of TNF- $\alpha$. JNK is mainly known to regulate the phosphorylation of c-Jun, which regulates the production of more c-Jun, along with other genes (30). Inflammatory cytokines induce the expression of c-Jun; however, the specific mechanism of how the inflammatory cytokines control the overexpression of c-Jun requires further research.

Previous studies reported that the cytokine TGF- $\beta$ can delay and repair IDD by upregulating ACAN and COL2A 1 expression, 
A
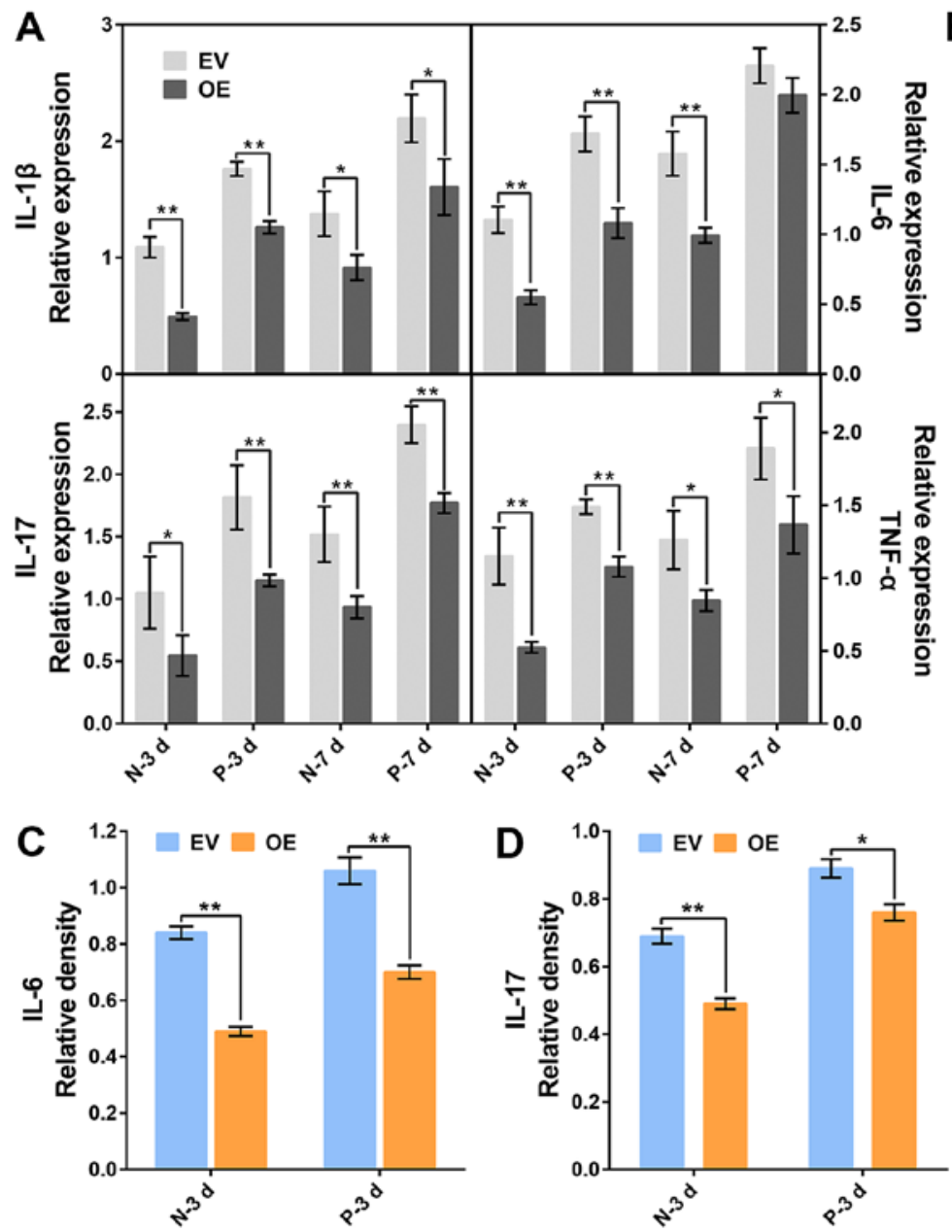

$\mathbf{F}$

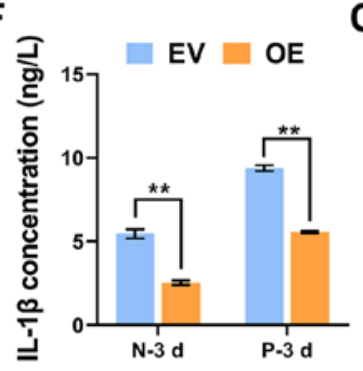

G

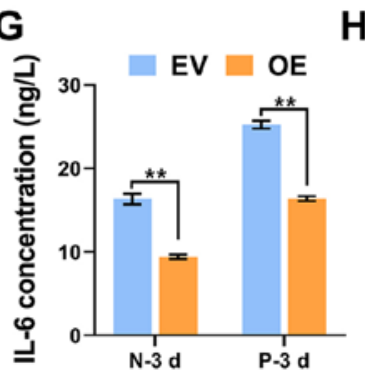

B
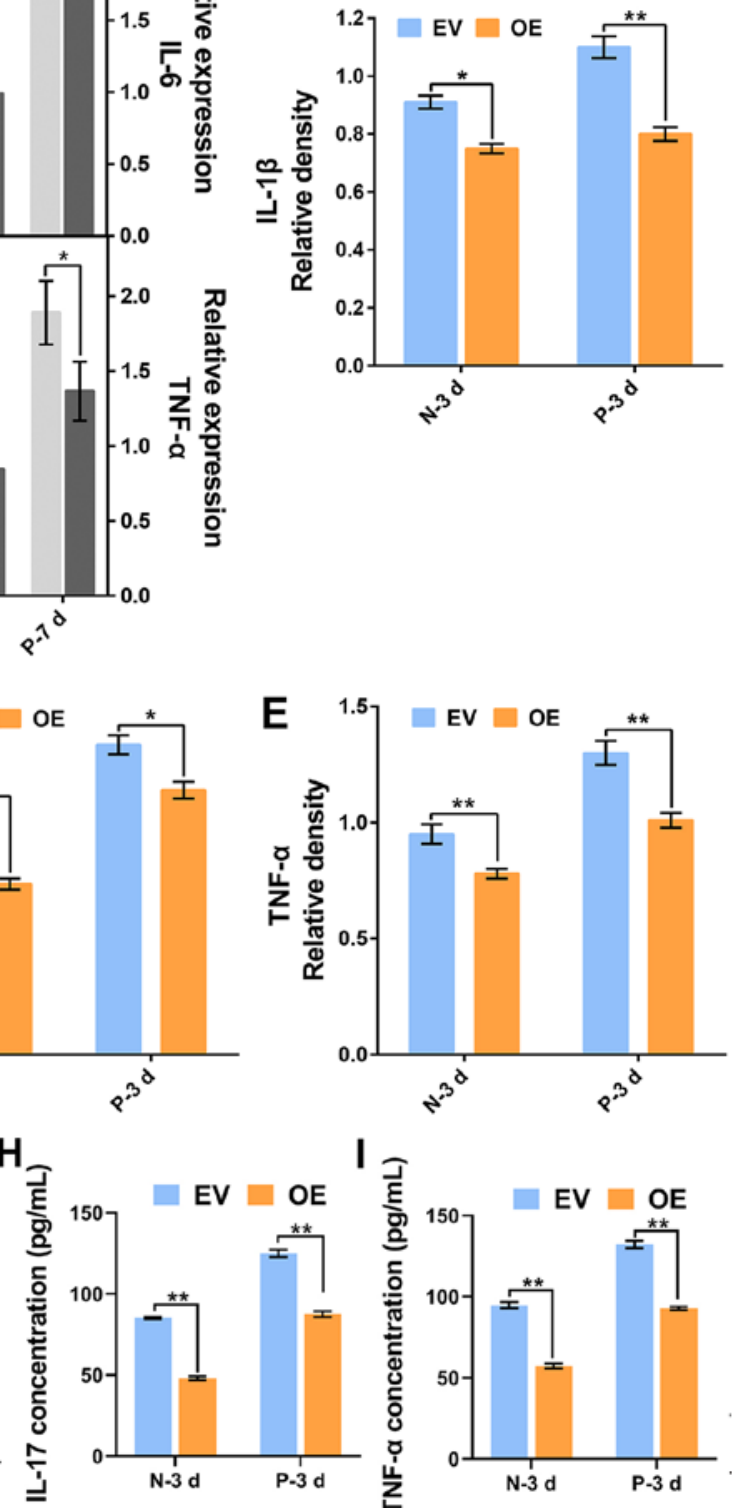

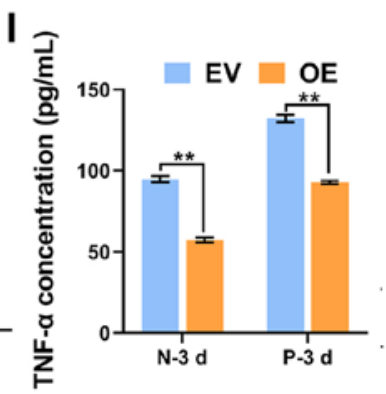

Figure 3. Expression of inflammatory cytokines in normal and degenerative NP cells followingc-Jun overexpression. (A) mRNA expression of inflammatory cytokines were detected by reverse transcription-quantitative PCR in cells cultured for 3 and 7 days. (B-E) Relative density of (B) IL-1 $\beta$, (C) IL-6, (D) IL-17 and (E) TNF- $\alpha$ was analyzed by ImageJ. (F-I) Concentrations of IL-1 $\beta$, IL-6, IL-17 and TNF- $\alpha$ in cell supernatant were analyzed by ELISA kit. *P $<0.05$, ${ }^{* *} \mathrm{P}<0.01$. EV, empty vector-transfected cells; OE, c-Jun-overexpressed cells; N-3 d, -7 d, normal cells were cultured for 3 days or 7 days; D-3 d, -7 d, degenerative cells were cultured for 3 days or 7 days.

therefore promoting NP cell proliferation and increasing ECM synthesis $(11,31,32)$. Furthermore, the proto-oncogene $c-m y c$ serves an active role in NP cell proliferation and cycle progression under TGF- $\beta$ stimulation (33). Interestingly, c-Jun belongs to proto-oncogene family of nuclear transcription factors, and it forms a complex with promoters of different genes to regulate transcription (23). Numerous growth-related cytokines contain 12-O-tetradecanoylphorbol-13-acetate response element (TRE) binding sites, including basic fibroblast growth factor and TGF- $\beta$, the dephosphorylated AP- 1 is able to recognize the TRE binding sites and initiate transcription of these cytokines (34). The Smad3-Smad4 heterodimeric complex cooperate with c-Jun/c-Fos to induce transcriptional activation in response to TGF- $\beta(21,35)$. Significant increase in TGF- $\beta$ mRNA and protein expression was observed in NP cells following c-Jun overexpression, and an upregulation was also observed for the cytokines TIMP-3, ACAN and COL2A1, which are associated with amelioration of IDD. It has been reported that TIMP3 can increase synthesis of ECM (36). COL2A1 and ACAN, which act as main components of matrix of NP (37), are essential to maintaining the integrity of IVD (4). The results from the present study suggested that c-Jun was positively related to IDD in NP cells, and that TGF- $\beta$ may act as a key regulator in c-Jun signaling pathway. Hiyama et al (38) reported that when NP cells are stimulated by TGF- $\beta$, there is a concomitant increase in c-Jun expression 


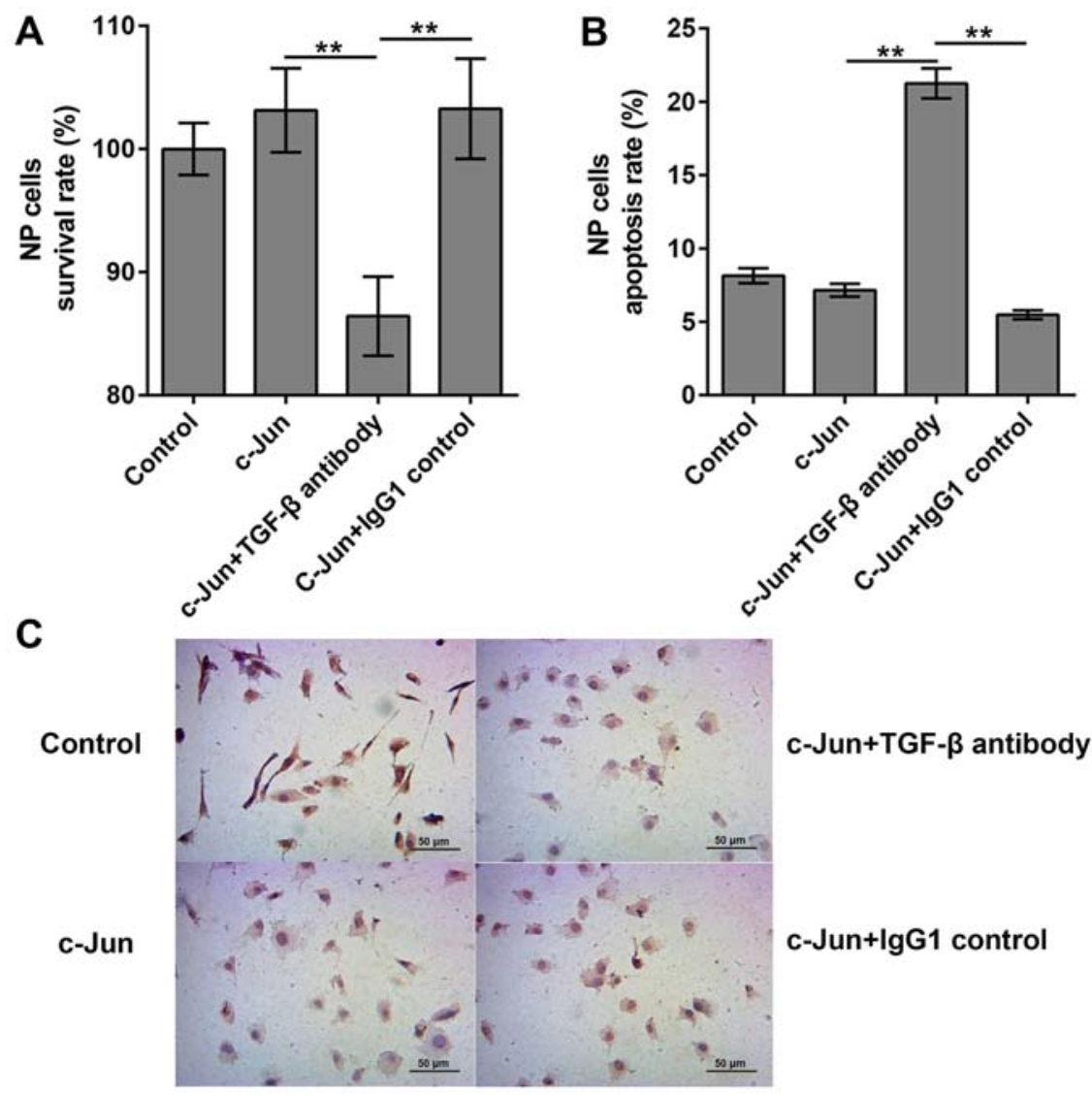

Figure 4. Proliferation and apoptosis rates of NP cells transfected with c-Jun after treatment with TGF- $\beta$ antibody. (A) Cell Counting Kit-8 assay showed a significant decrease in cell survival rates after treatment with TGF- $\beta$ antibody. (B and C) TUNEL assay showed an increase in cell apoptosis after treatment with TGF- $\beta$ antibody. Red represents TUNEL stained positive cells and blue is the negative control. ${ }^{* *} \mathrm{P}<0.01$. Scale, bar, $50 \mu \mathrm{m}$. Control indicated empty vector-transfected cells. IgG1 was used as a control for TGF- $\beta$ antibody. TGF- $\beta$, transforming growth factor- $\beta$; NP, nucleus pulposus.

and activity (38). In the present study, when patients' NP cells were transduced to overexpress c-Jun, cell survival rate was increased and apoptosis rate was decreased. However, there was no significant difference between the control group and the overexpressed c-Jun group. Treatment with antibody against TGF- $\beta$ significantly increased the rate of apoptosis and decreased the survival rate, suggesting that c-Jun might promote cell proliferation and inhibit cell apoptosis, thereby minimizing the function of c-Jun after inhibition of TGF- $\beta$. This result was consistent with previous studies showing that c-Jun promotes cell proliferation and protects cells from apoptosis (39-42).

In the present study, c-Jun increased the expression of TGF- $\beta$, TIMP-3, ACAN and COL2A1 which may promote ECM synthesis, and suppressed the inflammatory response by decreasing the expression of TNF- $\alpha$, IL-1 $\beta$, IL-6 and IL-17. Furthermore, c-Jun facilitated cell proliferation and decreased cell apoptosis by upregulating TGF- $\beta$ expression. These results suggested that c-Jun may alleviate IDD by regulating TGF- $\beta$. The results from this study allowed a better understanding of the molecular mechanisms of IDD, which may help the development of novel treatment of IDD disease. To the best of our knowledge, only a few studies have elucidated the role of c-Jun in IDD or additional degenerative diseases. The present study evaluated the effects of c-Jun on NP cells in vitro; however, whether c-Jun could delay disc degeneration in vivo, or directly interact with TGF- $\beta$ remain unknown. Further investigation is therefore required.

\section{Acknowledgements}

Not applicable.

\section{Funding}

No funding was received.

\section{Availability of data and materials}

All data generated or analyzed during this study are included in this published article.

\section{Authors' contributions}

ML performed the experiments, collected the results and wrote the manuscript. KW, SL, KZ, WH and XW contributed to data analysis and manuscript revision. $\mathrm{CY}$ conceived the study and contributed to reviewing/editing the manuscript. All authors read and approved the final manuscript.

\section{Ethics approval and consent to participate}

The study complied with the Declaration of Helsinki and was approved by the Institutional Review Board Ethics Committee of Union Hospital of Tongji Medical College. Written informed consent was obtained from each patient. 


\section{Patient consent for publication}

Not applicable.

\section{Competing interests}

The authors declare that they have no competing interests.

\section{References}

1. Freemont AJ: The cellular pathobiology of the degenerate intervertebral disc and discogenic back pain. Rheumatology (Oxford) 48: 5-10, 2009.

2. Hanaei S, Abdollahzade S, Khoshnevisan A, Kepler CK and Rezaei N: Genetic aspects of intervertebral disc degeneration. Rev Neurosci 26: 581-606, 2015.

3. Risbud MV and Shapiro IM: Role of cytokines in intervertebral disc degeneration: Pain and disc content. Nat Rev Rheumatol 10: 44-56, 2014.

4. Zheng L, Jianxiong S, Wu WK, Xin Y, Jinqian L, Guixing Q and Jiaming L: The role of leptin on the organization and expression of cytoskeleton elements in nucleus pulposus cells. J Orthop Res 31: 847-857, 2013.

5. Li Z, Liang J, WK WK, Yu X, Yu J, Weng X and Shen J: Leptin activates RhoA/ROCK pathway to induce cytoskeleton remodeling in nucleus pulposus cells. Int J Mol Sci 15: 1176-1188, 2014

6. Pattison ST, Melrose J, Ghosh P and Taylor TKF: Regulation of gelatinase-a (mmp-2) production by ovine intervertebral disc nucleus pulposus cells grown in alginate bead culture by transforming growth factor- $\beta$ and insulin like growth factor-1. Cell Biol Int 25: 679-689, 2001.

7. Gruber HE and Hanley EN Jr: Recent advances in disc cell biology. Spine (Phila Pa 1976) 28: 186-193, 2003.

8. Jianru W, Dessislava M, D Greg A, Zhaomin Z, Irving MS and Makarand VR: TNF- $\alpha$ and IL-1 $\beta$ promote a disintegrin-like and metalloprotease with thrombospondin type I motif-5-mediated aggrecan degradation through syndecan-4 in intervertebral disc. J Biol Chem 286: 39738-39749, 2011.

9. Wang WJ, Yu XH, Wang C, Yang W, He WS, Zhang SJ, Yan YG and Zhang J: MMPs and ADAMTSs in intervertebral disc degeneration. Clin Chim Acta 448: 238-246, 2015

10. Vo NV, Hartman RA, Yurube T, Jacobs LJ, Sowa GA and Kang JD: Expression and regulation of metalloproteinases and their inhibitors inintervertebral disc aging and degeneration. Spine J 13: 331-341, 2013

11. Jin H, Shen J, Wang B, Wang M, Shu B and Chen D: TGF- $\beta$ signaling plays an essential role in the growth and maintenance of intervertebral disc tissue. FEBS Lett 585: 1209-1215, 2011.

12. Feng C, Liu H, Yang M, Zhang Y, Huang B and Zhou Y: Disc cell senescence in intervertebral disc degeneration: Causes and molecular pathways. Cell Cycle 15: 1674-1684, 2016.

13. Angel P and Karin M: The role of Jun, Fos and the AP-1 complex in cell-proliferation and transformation. Biochim Biophys Acta 1072: 129-157, 1991.

14. Wisdom R, Johnson RS and Moore C: c-Jun regulates cell cycle progression and apoptosis by distinct mechanisms. EMBO J 18: 188-197, 1999.

15. Behrens A, Sibilia M and Wagner EF: Amino-terminal phosphorylation of c-Jun regulates stress-induced apoptosis and cellular proliferation. Nat Genet 21: 326-329, 1999.

16. Santos MM, Tannuri AC, Coelho MC, Gonçalves JD, Serafini S Da Silva LF and Tannuri U: Immediate expression of c-Fos and c-jun mRNA in a model of intestinal autotransplantation and ischemia-reperfusion in situ. Clinics (Sao Paulo) 70: 373-379, 2015

17. Behrens A, Sibilia M, David JP, Möhle-Steinlein U, Tronche F, Schütz G and Wagner EF: Impaired postnatal hepatocyte proliferation and liver regeneration in mice lacking $\mathrm{c}$-jun in the liver EMBO J 21: 1782-1790, 2002.

18. Behrens A, Haigh J, Mechta-Grigoriou F, Nagy A, Yaniv M and Wagner EF: Impaired intervertebral disc formation in the absence of Jun. Development 130: 103-109, 2003.

19. Pfirrmann CW, Metzdorf A, Zanetti M, Hodler J and Boos N: Magnetic resonance classification of lumbar intervertebral disc degeneration. Spine (Phila Pa 1976) 26: 1873-1878, 2001.

20. Livak KJ and Schmittgen TD: Analysis of relative gene expression data using real-time quantitative PCR and the 2(-Delta Delta $\mathrm{C}(\mathrm{T})$ ) method. Methods 25: 402-408, 2001.
21. Zhang Y, Feng XH and Derynck R: Smad3 and Smad4 cooperate with c-Jun/c-Fos to mediate TGF-beta-induced transcription. Nature 394: 909-913, 1998

22. Ye N, Ding Y, Wild C, Shen Q and Jia Z: Small molecule inhibitors targeting activator protein 1 (AP-1). J Med Chem 57: 6930-6948, 2014.

23. Tolonen J, Grönblad M, Virri J, Seitsalo S, Rytömaa T and Karaharju E: Oncoprotein c-Fos and c-Jun immunopositive cells and cell clusters in herniated intervertebral disc tissue. Eur Spine J 11: 452-458, 2002.

24. Monchaux M, Forterre S, Spreng D, Karol A, Forterre F and Wuertz-Kozak K: Inflammatory processes associated with canine intervertebral disc herniation. Front Immunol 8: 1681, 2017.

25. Liu XG, Hou HW and Liu YL: Expression levels of IL-17 and TNF- $\alpha$ in degenerated lumbar intervertebral discs and their correlation. Exp Ther Med 11: 2333-2340, 2016.

26. Liu Y, Du Y, Wang H, Li D and Feng WH: Porcine reproductive and respiratory syndrome virus (PRRSV) up-regulates IL-8 expression through TAK-1/JNK/AP-1 pathways. Virology 506: 64-72, 2017.

27. Shaulian E and Karin M: AP-1 as a regulator of cell life and death. Nat Cell Biol 4: E131-E136, 2002.

28. Li JK, Nie L, Zhao YP, Zhang YQ, Wang X, Wang SS, Liu Y, Zhao $\mathrm{H}$ and Cheng L: IL-17 mediates inflammatory reactions via p38/c-Fos and JNK/c-Jun activation in an AP-1-dependent manner in human nucleus pulposus cells. J Transl Med 14: 77, 2016.

29. Duval H, Mbatchi SF, Grandadam S, Legendre C, Loyer P, Ribault C, Piquet-Pellorce C, Guguen-Guillouzo C, Boudjema K and Corlu A: Reperfusion stress induced during intermittent selective clamping accelerates rat liver regeneration through JNK pathway. J Hepatol 52: 560-569, 2010.

30. Koba S, Pakala R, Watanabe T, Katagiri T and Benedict CR: Vascular smooth muscle proliferation-a protein kinase stimulated by UV light and Ha-Ras that binds and phosphorylates the c-Jun activation domain. J Am Coll Cardiol 34: 1644-1651, 1999.

31. Lee YJ, Kong MH, Song KY, Lee KH and Heo SH: The relation between Sox9, TGF-beta1, and proteoglycan in human intervertebral disc cells. J Korean Neurosurg Soc 43: 149-154, 2008.

32. Wang SL, Yu YL, Tang CL and Lv FZ: Effects of TGF- $\beta 1$ and IL-1 $\beta$ on expression of ADAMTS enzymes and TIMP-3 in human intervertebral disc degeneration. Exp Ther Med 6: $1522-1526,2013$

33. Nakai T, Mochida J and Sakai D: Synergistic role of c-Myc and ERK1/2 in the mitogenic response to TGF beta-1 in cultured rat nucleus pulposus cells. Arthritis Res Ther 10: R140, 2008

34. Iwata A, Masago A and Yamada K: Expression of basic fibroblast growth factor mRNA after transient focal ischemia: Comparison with expression of c-fos, c-jun, and hsp 70 mRNA. J Neurotrauma 14: 201-210, 1997

35. Qing J, Zhang Y and Derynck R: Structural and functional characterization of the transforming growth factor-beta-induced Smad3/c-Jun transcriptional cooperativity. J Biol Chem 275: 38802-38812, 2000.

36. Yan L, Kang L, Han X, Mao C, Kai Z, Zhao T and Jie Z: The imbalance between TIMP3 and matrix-degrading enzymes plays an important role in intervertebral disc degeneration. Biochem Biophys Res Commun 469: 507-514, 2016.

37. Chung SA, Khan SN and Diwan AD: The molecular basis of intervertebral disk degeneration. Orthop Clin North Am 34: 209-219, 2003.

38. Hiyama A, Gogate SS, Gajghate S, Mochida J, Shapiro IM and Risbud MV: BMP-2 and TGF-beta stimulate expression of beta 1,3-glucuronosyl transferase 1 (GlcAT-1) in nucleus pulposus cells through AP1, TonEBP, and Sp1: Role of MAPKs. J Bone Miner Res 25: 1179-1190, 2010.

39. Gaudet P, Livstone MS, Lewis SE and Thomas PD: Phylogenetic-based propagation of functional annotations within the gene ontology consortium. Brief Bioinform 12: 449-462, 2011.

40. Qian Z, Li Y, Chen J, Li X and Gou D: MiR-4632 mediates PDGFBB-induced proliferation and anti-apoptosis of human pulmonary artery smooth muscle cells via targeting cJUN. Am J Physiol Cell Physiol 313: C380-C391, 2017.

41. Khachigian LM, Fahmy RG, Zhang G, Bobryshev YV and Kaniaros A: c-Jun regulates vascular smooth muscle cell growth and neointima formation after arterial injury. Inhibition by a novel DNA enzyme targeting c-Jun. J Biol Chem 277: 22985-22991, 2002.

42. Kappelmann M, Bosserhoff A and Kuphal S: AP-1/c-Jun transcription factors: Regulation and function in malignant melanoma. Eur J Cell Biol 93: 76-81, 2014.

This work is licensed under a Creative Commons Attribution-NonCommercial-NoDerivatives 4.0 International (CC BY-NC-ND 4.0) License. 